\title{
Motion Region Detection and Pattern Extraction Algorithm of a Motion Image Based on a Radar Sensor
}

\author{
Jinseop Lee*
}

\begin{abstract}
In this paper, a motion region detection and pattern extraction algorithm is proposed for motion recognition based on the image as the distance changes between the radar sensor and human motion. This algorithm, which was developed by combining the characteristics of the radar motion image and image processing techniques, compensates for the discontinuous data that can occur when removing clutter for motion data detection and extracts motion patterns applicable to the recognition learning field. According to the motion data measured by a radar sensor, the algorithm performance is verified by an experiment.
\end{abstract}

Key Words: Machine Learning, Motion Detection, Motion Recognition, Pattern Extraction, Radar Image Processing.

\section{INTRODUCTION}

Existing motion recognition technology is conducted by capturing human movements through visual images using cameras. Recently, Google has implemented motion recognition using a chip through "Soli" [1]. Motion recognition using this miniaturized radar chip technology can be applied to a wide range of applications, including internet of things and wearable devices. Among all the motion recognition technologies, the radarapplied motion recognition can be identified in both natural environment and non-visual situations. Radar for motion recognition is mainly used for ultra-wideband (UWB), frequency-modulated continuous wave (FMCW) radar. Comparing the two radars, the UWB radar has a lower transmission power and a shorter coverage, but its resolution is more precise and its response rate is higher than that of the FMCW radar.

A typical technique for motion recognition using a radar sensor is to recognize pattern by extracting micro-Doppler based on a CW radar [2]. Based on these techniques, motion is recognized through the amplitude $[3,4]$ of the received signal reflected in the motion or through the Doppler information $[5,6]$ of the received signal. Recognition studies based on machine learning and deep learning are actively being pursued [4, 6-8].

Motion is also recognized through images resulting from the changes in the distance between the radar sensor and human motion [9]. First, the radar-received signals are accumulated, and signal processing is performed to remove the clutter in a two (pulse frame-sample)-dimensional image. Second, feature extraction and learning of the motion image using the histogram of oriented gradient/support vector machine (SVM) are conducted. In [9], a good recognition performance was verified with the data of actual measured human motion. However, motion recognition based on distance changes is essential to the process to reject a fixed clutter. This process may remove some of the motion data. That is, when recognizing motion that requires sophistication, performance may be degraded.

Manuscript received April 12, 2019 ; Revised June 26, 2019 ; Accepted August 5, 2019. (ID No. 20190412-018J)

Hanwha Systems, Pangyo, Korea.

"Corresponding Author: Jinseop Lee (e-mail: jinseop.llee@gmail.com)

This is an Open-Access article distributed under the terms of the Creative Commons Attribution Non-Commercial License (http://creativecommons.org/licenses/by-nc/4.0) which permits unrestricted non-commercial use, distribution, and reproduction in any medium, provided the original work is properly cited.

(c) Copyright The Korean Institute of Electromagnetic Engineering and Science. 
In this paper, a motion region extraction and pattern extraction algorithm is proposed for motion recognition based on the image as the distance changes between the radar sensor and human motion. This algorithm, which was developed by combining the characteristics of radar motion images and image processing techniques, compensates for the discontinuous data that can occur when removing clutter for motion data extraction and extracts motion patterns applicable to the recognition learning field. Using the motion data measured by the radar sensor, the algorithm performance is verified by an experiment.

This paper is organized as follows. Section II discusses the concept and the result of clutter processing for motion data extraction. Section III describes the proposed algorithm step by step based on the results in Section II. Section IV presents the processing result for the algorithm in Section III. The paper is concluded in Section V.

\section{ClutTer Removal Processing FOR}

\section{MOTION DATA EXTRACTION}

Clutter removal is recognized as a change in the distance between the radar sensor and human motion. This process of removing leakage signals from radar sensors and the effects of fixed clutter such as on objects [9] is essential. By performing the moving average filter along the pulse axis, the effect of the clutter can be removed, and only motion data are extracted. The clutter removal process can be expressed as

$$
x_{p, k}=x_{p, k}-\frac{1}{M} \sum_{j=0}^{M} x_{p-j, k},
$$

where $x_{p, k}$ is the data of the received motion image, and $p$ and $k$ are the pulse frame and the sample index of a single pulse, respectively. The raw data and clutter removal processing results are shown in Fig. 1.

The $x$-axis and $y$-axis in Fig. 1 represent the pulse frame and the samples of a single pulse, respectively. Motion data can be extracted by removing the clutter, as shown in Fig. 1. However, discontinuous motion data may occur when the motion is maintained at the same distance for a while or during the state of motion transition. That is, compensation is required through

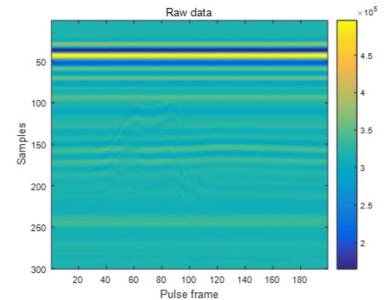

(a)

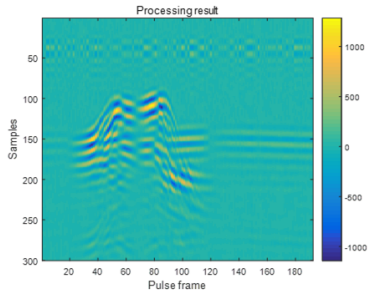

(b)
Fig. 1. Result of the clutter removal process: (a) raw data and (b) clutter removal. the tendency of the adjacent motion regions.

\section{PROPOSED MOTION REGION DETECTION AND PATTERN EXTRACTION ALGORITHM}

In this section, the motion region detection and pattern extraction algorithm are proposed for motion recognition based on the distance changes between the radar sensor and human motion. This algorithm can extract the pattern of motion image and compensate for the discontinuous motion image in Fig. 1. The flow chart of the algorithm is illustrated in Fig. 2.

\section{Region of Interest (ROI)}

ROI extraction is performed to remove leakage signal from the radar sensor. In particular, leakage signal data that have not been removed from the signal processing can degrade the performance of the algorithm. In Fig. 1(a), the amplitude of the signal is strong at the 40th-50th sample intervals. Therefore, it can be estimated as a leakage signal.

\section{Binarization Process}

The binarization process extracts the motion data and performs binarization. The motion data are extracted by processing the average of the image and the threshold value of each sample. The flow chart of the binarization process is shown in Fig. 3.

The equation of the threshold for the binarization process is as follows:

$$
\begin{aligned}
& T_{a}=k_{a} \cdot a_{a} \\
& T_{s}=k_{s} \cdot a_{s},
\end{aligned}
$$

where $a_{a}$ and $a_{s}$ represent the average value of the image and of each equivalent sample, respectively, $k_{a}$ and $k_{s}$ are the

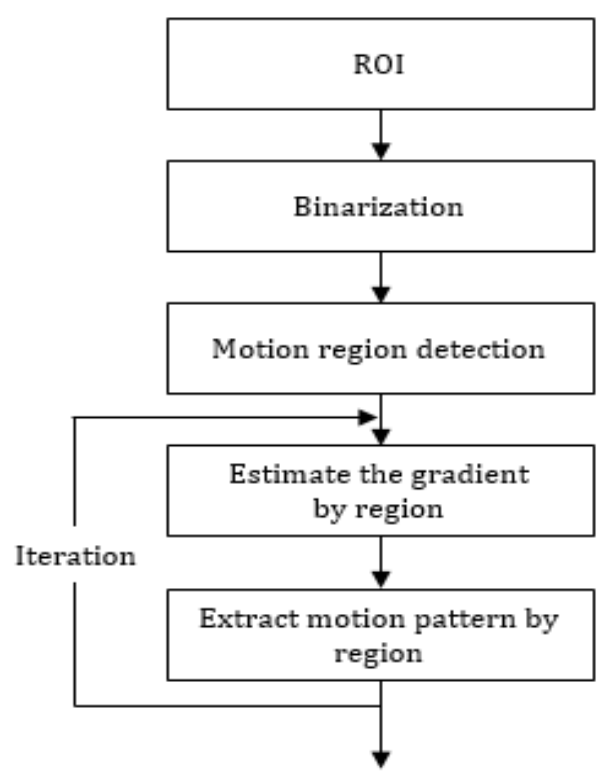

Fig. 2. Flow chart of the pattern extraction algorithm. 
threshold scale factors, and $T_{a}$ and $T_{s}$ are the calculated threshold value. As the amplitude of the motion data is greater than the average value of the image or sample, extracting the motion data is possible.

\section{Motion Region Detection}

To extract the pattern of the motion correctly, only the detection of the motion region is required. The flow chart of the motion region detection is illustrated in Fig. 4.

Based on the data after the binarization process, the motion region is detected through the density of motion data. The region for determining the density is expressed as a window region. To improve the processing speed of the calculation, the density of the region is calculated while moving to the step size $\left(l_{r, s t e p}, l_{c, s t e p}\right)$. The conditional inequality for determining density is

$$
C_{d}>k_{d} \cdot C_{w},
$$

where $k_{d}$ is the ratio of the window region size and should be properly set to the motion data and the resolution of the radar sensor. If Eq. (4) is not satisfied, the data are removed. The concept of motion region detection is presented in Fig. 5.

Data are maintained or removed by calculating the density of the window region.

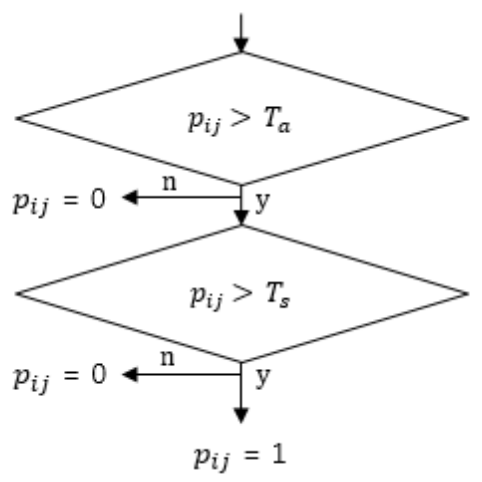

Fig. 3. Flow chart of the binarization process.

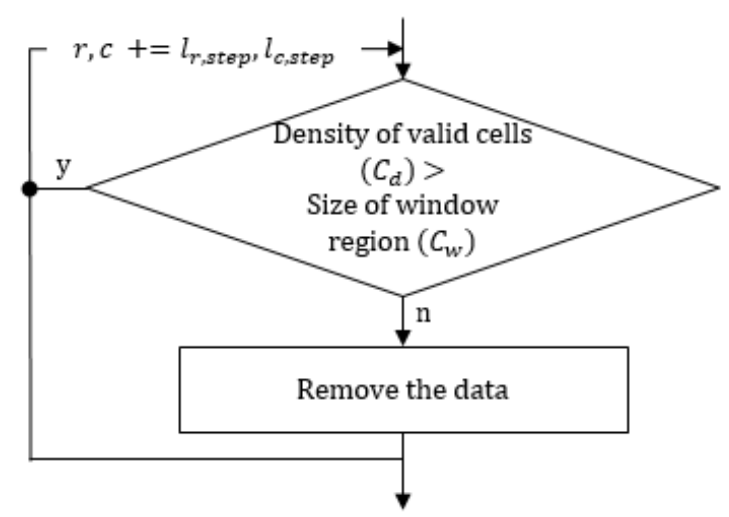

Fig. 4. Flow chart of the motion region detection.

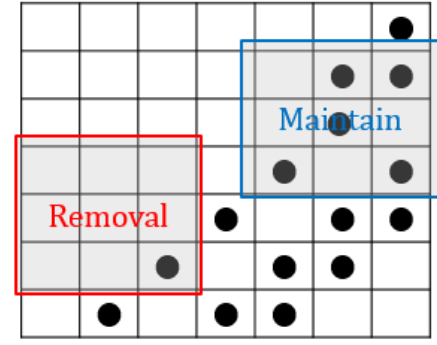

Fig. 5. Motion region detection concept.

\section{Gradient Estimation by Region}

The detected motion region is divided into small regions, and the gradient for each region is estimated. The acquired motion data are an image of a time-range axis that accumulates into a radar pulse. Therefore, a gradient in the vertical direction does not occur. The gradient estimation uses a kernel-type method using two-dimensional (2D) filtering instead of a general image processing method. Fig. 6 shows the shape of the gradient kernel.

Fig. 6(a) shows the gradient of the kernel matrix, and Fig. 6(b) presents the direction. The vertical gradient is not taken into account because of the measured radar motion characteristics. The kernel size is determined by the size of the region to be processed. The similarity between the region and the kernels is calculated through $2 \mathrm{D}$ filtering. Fig. 7 shows the flow chart that estimates the gradient by region based on the kernel.

As shown in Fig. 7, the gradient of the region is estimated by calculating the similarity with the kernel. The two conditions in Fig. 7 are as follows:

Condition 1: no similarity for all-kernels

Condition 2: two or more of the most similar calculated values are the same

$$
\left[\begin{array}{lll}
0 & 0 & 0 \\
1 & 1 & 1 \\
0 & 0 & 0
\end{array}\right]\left[\begin{array}{lll}
0 & 0 & 1 \\
0 & 1 & 0 \\
1 & 0 & 0
\end{array}\right]\left[\begin{array}{lll}
1 & 0 & 0 \\
0 & 1 & 0 \\
0 & 0 & 1
\end{array}\right]
$$

(a)

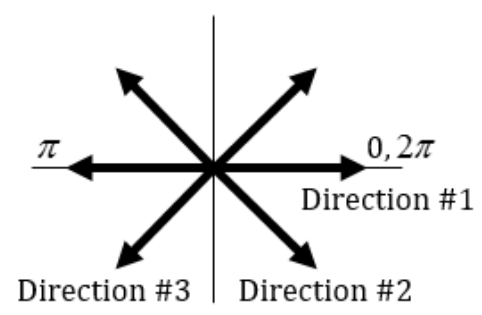

(b)

Fig. 6. Shape of the gradient kernel: (a) gradient kernel matrix and (b) direction of the gradient kernel. 


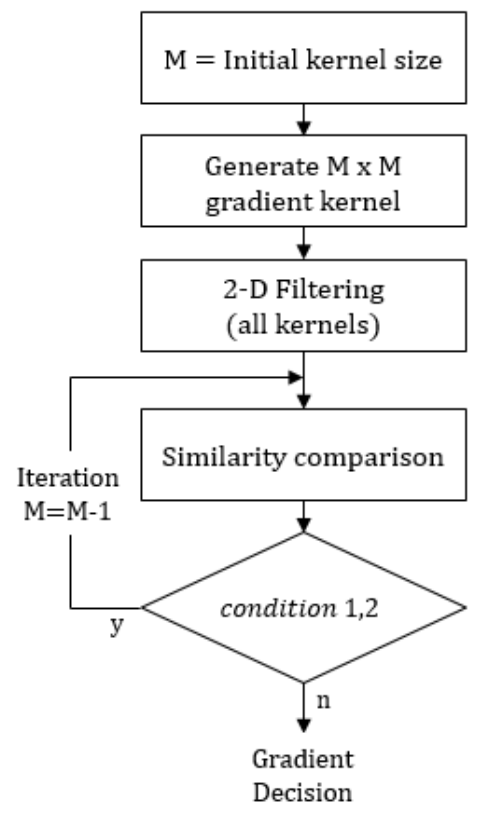

Fig. 7. Gradient estimation flow chart by region.

If the result of calculating the similarity with the $M_{k} \times$ $M_{k}$ kernel is included in the above condition 1 or 2 , the $M_{k-1} \times M_{k-1}$ kernel will repeat the similarity calculation to estimate the gradient.

\section{Pattern Extraction by Region}

To estimate and extract patterns for each region, motion patterns are estimated by comparing the gradients between the region and the adjacent region. The flow chart for the gradient estimation is presented in Fig. 8.

The estimation of the gradient of the region consists of two stages. The first stage compares the gradients of the adjacent regions in the same direction. For example, in Fig. 9, if the gradient in the red square region $R_{i j}$ is in direction 3 , the gradient in regions $R_{i+1, j-1}$ and $R_{i-1, j+1}$ in direction 3 will be compared. If all three regions have the same direction 3 , the gradient is determined in direction 3 .

However, if any of the compared gradient is different as shown in Fig. 9, then the gradient in region $R_{i j}$ is most likely incorrectly estimated. This condition can degrade performance when extracting motion. Therefore, in this case, the gradient of the adjacent regions should be compared. Fig. 10 shows the

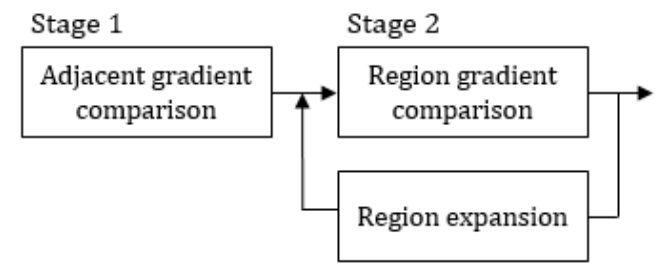

Fig. 8. Flow chart of the pattern extraction by region.

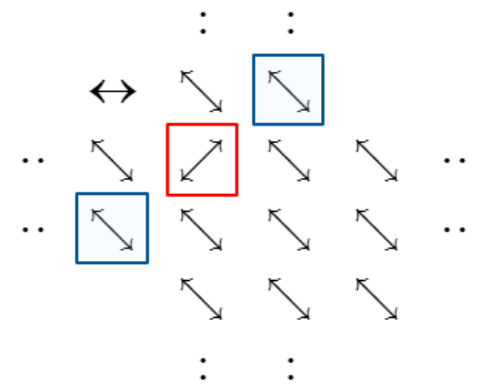

Fig. 9. Concept of the first stage.

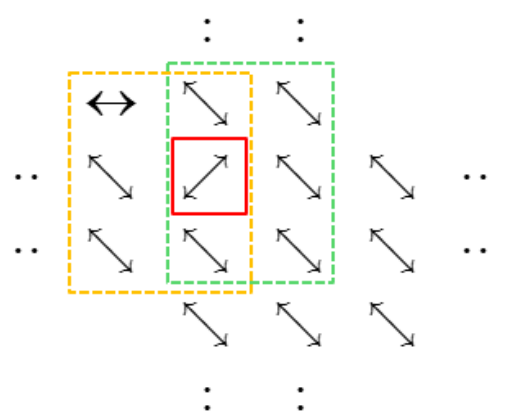

Fig. 10. Concept of the second stage.

concept of the second stage in estimating the patterns from the gradients in the adjacent regions.

In Fig. 10, the yellow box is the left region, and the green box is the right region. The gradient of the red box $R_{i j}$ is estimated by determining the dominant gradient of both regions. As direction 2 is dominant in both regions, $R_{i j}$ is determined in direction 2. If there is no dominant gradient in the region, the comparison region will be extended, as shown in Fig. 11.

Then, the motion pattern of the region is simplified based on the estimated gradient on the left and the right. A pattern matrix of a $5 \times 5$ size is shown in Fig. 12 .

The type names in Fig. 12 are in the left and right directions. For example, if the dominant gradient is direction 2 in the left region and the dominant gradient is direction 3 in the right region, then the pattern type is "Type 23". The image of the motion region is replaced by a pattern matrix based on the estimated gradient.

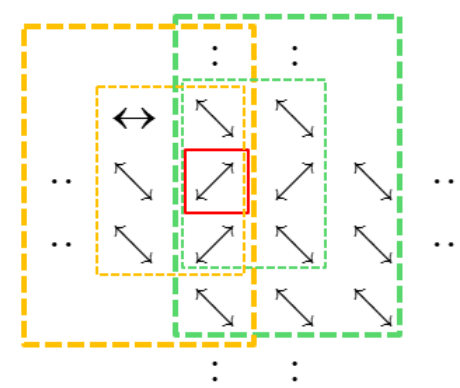

Fig. 11. Region expansion in the second stage. 


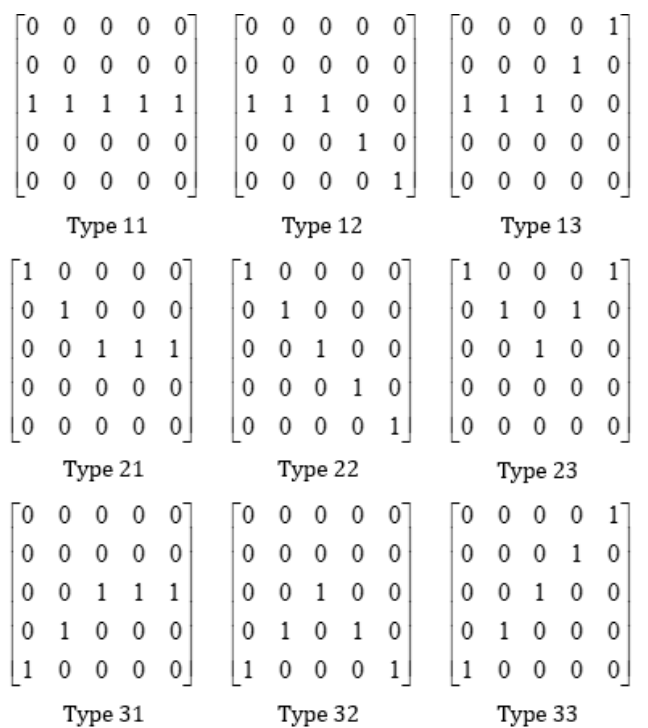

Fig. 12. A $5 \times 5$ pattern matrix.

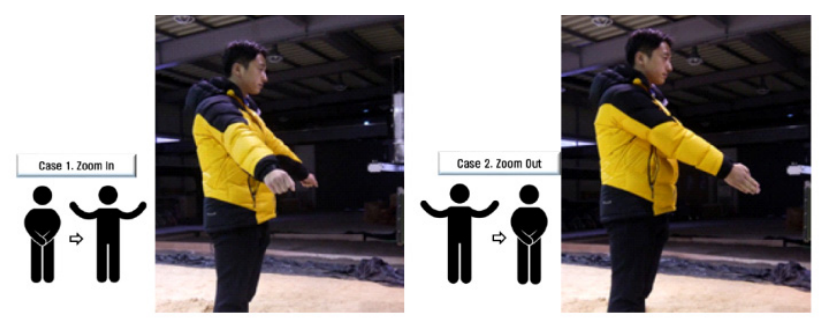

Fig. 13. Two motions in the experiment.

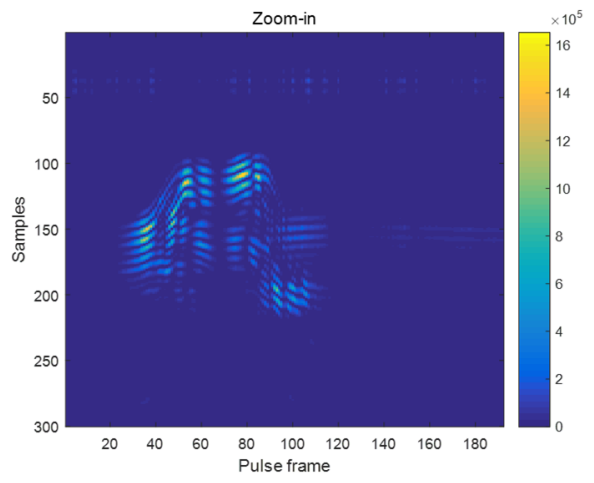

(a)

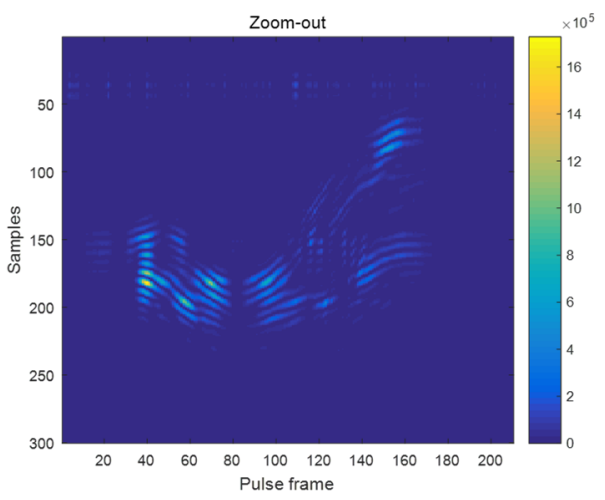

(b)

Fig. 14. Signal processing result: (a) zoom-in and (b) zoom-out.

\section{EXPERIMENTAL RESULT}

To verify the performance of the proposed algorithm, we experiment with the measured human motion images based on the radar sensor. The two measured motions are shown in Fig. 13. The signal processing algorithm results in [9] on motion are shown in Fig. 14.

The UWB radar sensor is used, and the sampling interval is $0.005 \mathrm{~m}$. The ROI is processed to acquire an image after the 70th sample to remove the leakage signal in Fig. 1. Then, binarization is performed through the threshold processing based on the average value of the image and each sample. $T_{a}$ and $T_{s}$ are obtained by analyzing the distribution of the measured radar image. The two times the average of $k_{a}$ and $k_{s}$, respectively, are appropriate. The binarization processing result of the zoomin motion image when $k_{a}$ and $k_{s}$ are 2 , respectively, is shown in Fig. 15.

Fig. 15 shows the extracted motion data after binarization. However, the non-motion data are incorrectly extracted in Fig. 15(b). These data are removed with the following motion region detection method. The result of the motion region detection using the $10 \times 10$ window region is presented in Fig. 16 . The step size of the row and column are respectively set to $1 / 2$ of

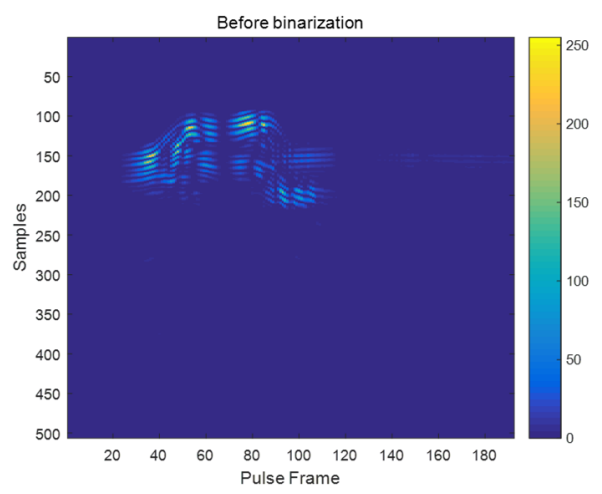

(a)

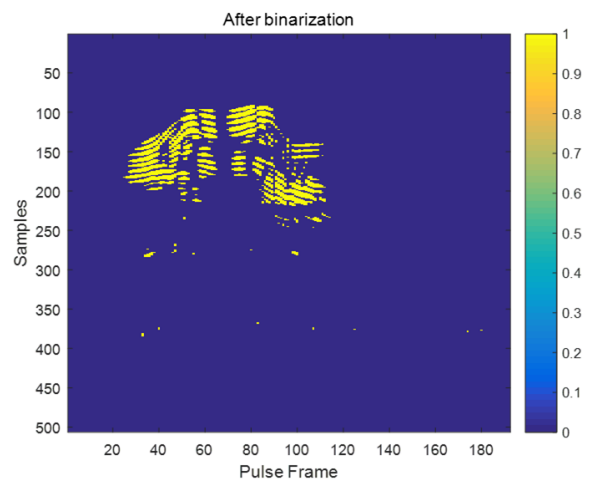

(b)

Fig. 15. Binarization processing result: (a) before binarization and (b) after binarization. 


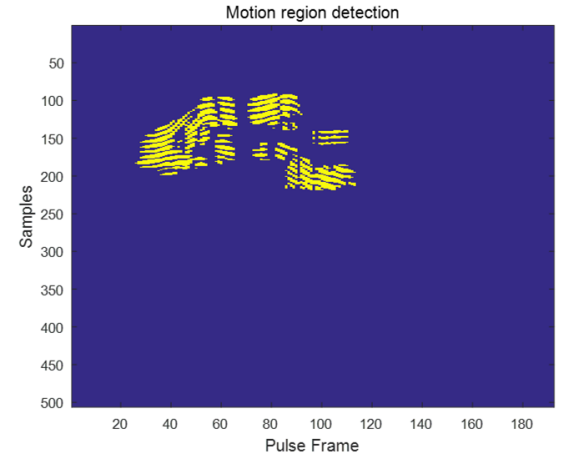

(a)

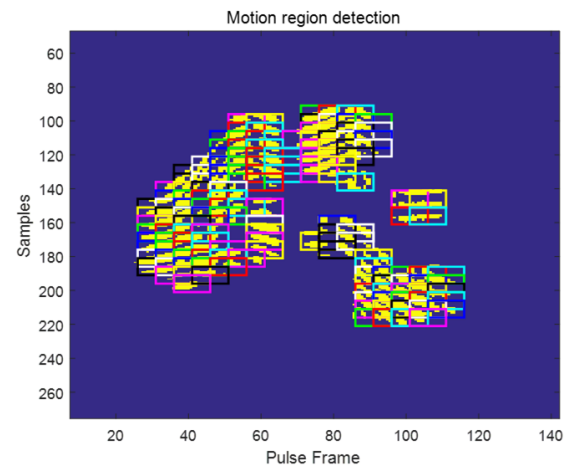

(b)

Fig. 16. Motion region detection result: (a) motion region detection and (b) display of valid motion region.

the region length, and density is set to $1 / 3$ of the window region. This process detects only the motion region and shows that the

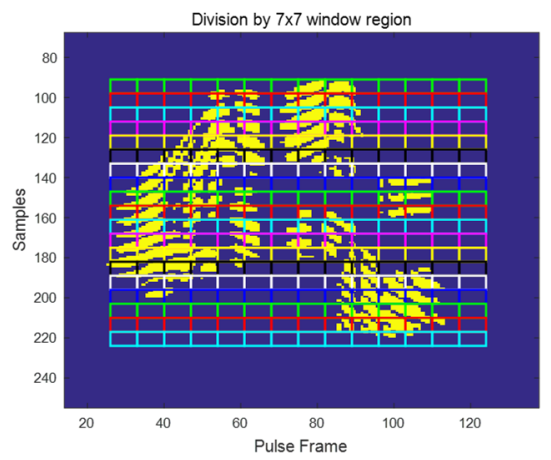

(a)

\begin{tabular}{|c|c|c|c|c|c|}
\hline 98 & $\theta$ & $\theta$ & $\theta$ & 11 & 11 \\
\hline & $\theta$ & (1) & 11 & 11 & 11 \\
\hline & $\theta$ & $\theta$ & 13 & 11 & 24 \\
\hline & $\theta$ & $\theta$ & 13 & 24 & 24 \\
\hline & $\theta$ & 11 & 13 & 11 & 24 \\
\hline & $\theta$ & 11 & 13 & 11 & 24 \\
\hline$\theta$ & 13 & 11 & 11 & 11 & 11 \\
\hline 11 & 13 & 11 & 24 & 11 & 11 \\
\hline 11 & 13 & 13 & 13 & 11 & 11 \\
\hline 11 & 13 & 13 & 13 & 11 & 11 \\
\hline 11 & 13 & 11 & 13 & 24 & 24 \\
\hline 11 & 13 & 13 & 13 & 11 & 11 \\
\hline 11 & 13 & 13 & 13 & 11 & 24 \\
\hline 11 & 1.3 & 13 & 11 & 11 & $\theta$ \\
\hline 11 & 11 & 11 & 0 & 0 & $\theta$ \\
\hline 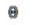 & 11 & 13 & $\theta$ & (๑) & a \\
\hline & $\theta$ & $\theta$ & $\theta$ & (8) & $\theta$ \\
\hline & $\theta$ & $\theta$ & $\theta$ & $\theta$ & $\theta$ \\
\hline$\theta$ & $\theta$ & $\theta$ & $\theta$ & $\theta$ & $\theta$ \\
\hline
\end{tabular}

\begin{tabular}{|c|c|c|c|c|}
\hline & & & & \\
\hline & 24. & 24 & $\theta$ & \\
\hline 13 & $\begin{array}{l}13 \\
{ }_{13}\end{array}$ & $\frac{24}{24}$ & $\stackrel{\theta}{\pi}$ & 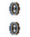 \\
\hline & & * & 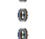 & a \\
\hline 11 & 24 & 24 & 要 & $\theta$ \\
\hline & 11 & 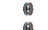 & ${ }_{11}^{11}$ & 11 \\
\hline & 24 & 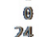 & 111 & 111 \\
\hline & 24 & 11 & 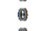 & $\theta$ \\
\hline & 13 & 24 & 111 & 111 \\
\hline & $\begin{array}{r}24 \\
24\end{array}$ & $\begin{array}{l}13 \\
24\end{array}$ & $\frac{24}{24}$ & 24 \\
\hline & 24 & & 78 & \\
\hline & $\begin{array}{r}24 \\
8\end{array}$ & $\frac{24}{24}$ & 11 & i11 \\
\hline
\end{tabular}

(b)

Fig. 17. Result of estimation of the gradient by region: division by the $7 \times 7$ window region and (b) gradient estimation result.

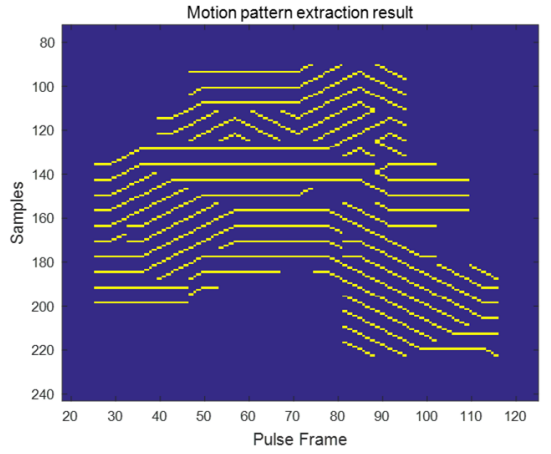

Fig. 18. Motion pattern extraction result.

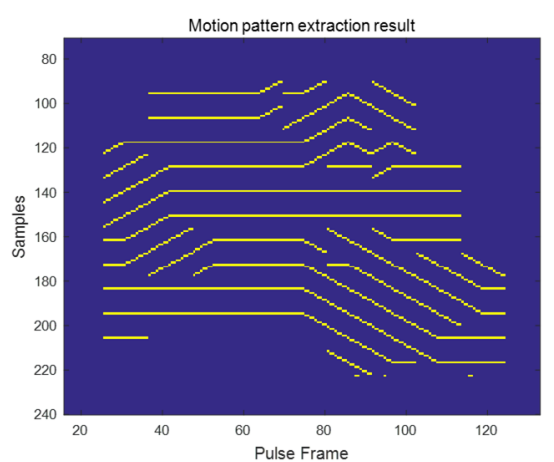

Fig. 19. Iterative motion pattern extraction result.

wrongly detected data in Fig. 15 are removed.

Based on the detected motion region, the region is divided for the gradient calculation by region. Fig. 17 shows the result of estimating the gradient by dividing it into a $7 \times 7$ matrix.

The results of estimating the gradient using the kernel matrix from $7 \times 7$ to $3 \times 3$ are shown in Fig. 17(b). The numbers in Fig. 17(b) are the direction shown in Fig. 6. The numbers 11, 24, and 13 in Fig. 15(b) are in directions 1-3, respectively. Fig. 18 shows the pattern extraction result based on the estimated gradient.

The existing motion image is simplified to one width of the image through pattern estimation and extraction. This process also compensates for the discontinuous interval motion data that occurs during the removal of the clutter in Fig. 1. If the algorithm is performed iteratively by expanding the region unit of the gradient, the motion image can be further simplified. The result of iteratively extracting the gradient window region to 7 $\times 7$ and $11 \times 11$ is shown in Fig. 19 .

The radar image depends on the speed and range of motion. Therefore, the window region size and the number of iterations should be determined by human motion and the sampling interval of the radar sensor. The pattern extraction results for the zoom-out motion are shown in Fig. 20.

The performance of the proposed algorithm is verified through a motion recognition experiment. Each motion uses 50 data sets for the experiment. Iteratively performing with the window region size of $7 \times 7$, the $11 \times 11$ window region ex- 


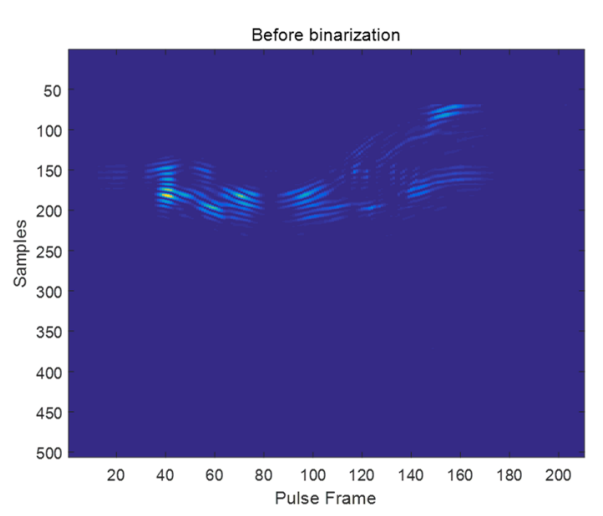

(a)

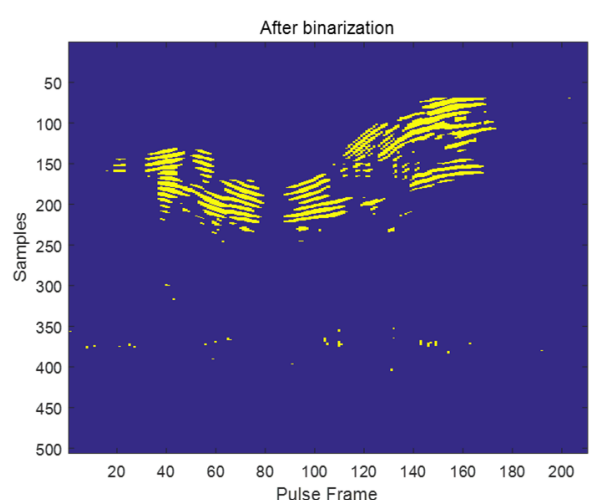

(b)

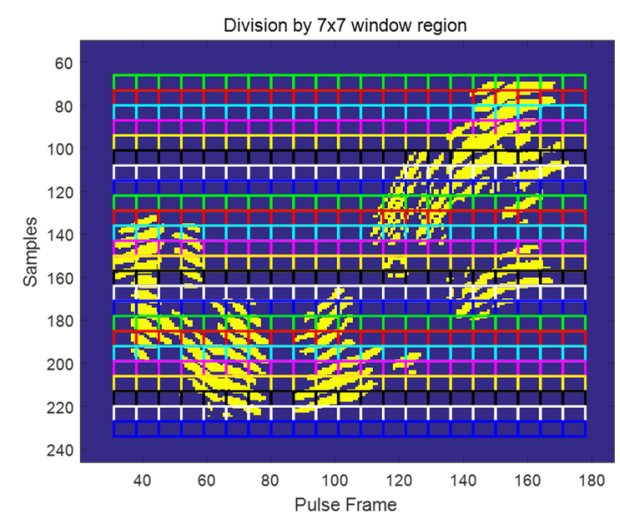

(c)

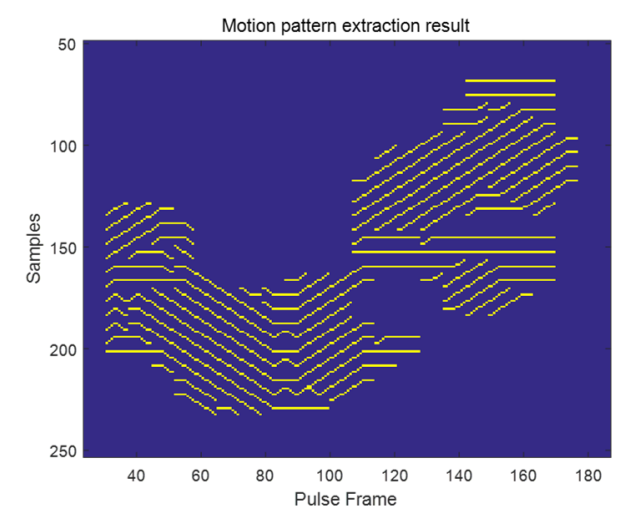

(d)

Fig. 20. Experimental results of the zoom-out motion: (a) raw data, (b) motion region detection, (c) division by the 7 $\times 7$ window region, and (d) motion pattern extraction result.
Table 1. Results of the motion recognition experiment

\begin{tabular}{lccc}
\hline & & \multicolumn{2}{c}{ Experimental result } \\
\cline { 3 - 4 } & & ZI & ZO \\
\hline Actual result & ZI & 1.00 & 0.00 \\
& ZO & 0.06 & 0.94 \\
\hline
\end{tabular}

tracted the patterns of two motions and recognized them through SVM learning. The k-fold cross-validation was applied to increase the statistical reliability of the experiment. The motion recognition experiment results are shown in Table 1.

The learning result of the zoom-in (ZI) motion shows a 100\% recognition rate and that of the zoom-out $(\mathrm{ZO})$ motion shows a $94 \%$ recognition rate.

\section{CONCLUSION}

A motion region detection and pattern extraction algorithm is proposed for motion recognition based on the image as distance changes between the radar sensor and human motion. This algorithm, which was developed by combining the characteristics of radar motion images and image processing techniques, compensates for the discontinuous data that can occur when removing clutter for motion data detection and extracts motion patterns that are applicable to the recognition learning field. Based on the motion data measured by the radar sensor, the algorithm performance is verified by an experiment. Although the performance is good in the experiment, the recognition performance can be further improved by performing the machine learning by applying hyperparameter learning and so on.

However, measuring the motion on the radar side distorts the image. Therefore, studies should recognize the motion of images that are distorted when measured on the side of the radar sensor. In future studies, we will examine the radar-based motion recognition based on deep learning, which has recently become popular.

\section{REFERENCES}

[1] J. Lien, N. Gillian, M. E. Karagozler, P. Amihood, C. Schwesig, E. Olson, H. Raja, and I. Poupyrev, "Soli: ubiquitous gesture sensing with millimeter wave radar," $A C M$ Transactions on Graphics (TOG), vol.35, no. 4, article no. 142, 2016.

[2] Y. Kim and B. Toomajian, "Hand gesture recognition using micro-Doppler signatures with convolutional neural network," IEEE Access, vol. 4, pp. 7125-7130, 2016.

[3] N. Ren, X. Quan, and S. H. Cho, "Algorithm for gesture recognition using an IR-UWB radar sensor," Journal of Computer and Communications, vol. 4, pp. 95-100, 2016. 
[4] J. Park and S. H. Cho, "IR-UWB radar sensor for human gesture recognition by using machine learning," in Proceedings of 2016 IEEE 18th International Conference on High Performance Computing and Communications; IEEE 14th International Conference on Smart City; IEEE 2nd International Conference on Data Science and Systems (HPCC/SmartCity/ DSS), Sydney, Australia, 2016, pp. 1246-1249.

[5] M. G. Amin, Z. Zeng, and T. Shan, "Hand gesture recognition based on radar micro-Doppler signature envelopes," 2018; https://arxiv.org/abs/1811.12467.

[6] Y. Kim and T. Moon, "Human detection and activity classification based on micro-Doppler signatures using deep convolutional neural networks," IEEE Geoscience and Remote Sensing Letters, vol. 13, no. 1, pp. 8-12, 2015.

Jinseop Lee

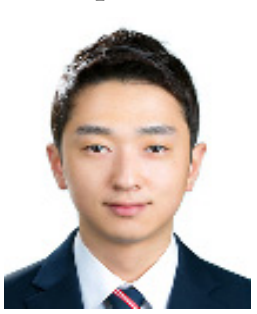

obtained his B.S. and M.S. degrees in electronic engineering from Konkuk University, Seoul, Korea, in 2014 and 2016, respectively. Since 2016, he has been a researcher at Hanwha Systems. His research interests include 2D/3D image detection/tracking, deep learning, speech recognition, and radar/image signal processing.
[7] S. Bhattacharya, B. Czejdo, and N. Perez, "Gesture classification with machine learning using Kinect sensor data," in Proceedings of 2012 3rd International Conference on Emerging Applications of Information Technology, Kolkata, India, 2012, pp. 348-351.

[8] S. Xiao, Z. Wang, and J. Folkesson, "Unsupervised robot learning to predict person motion," in Proceedings of 2015 IEEE International Conference on Robotics and Automation (ICRA), Seattle, WA, 2015, pp. 691-696.

[9] J. S. Lee and J. W. Yoon, "A study of motion recognition using IR-UWB radar," The Journal of Korean Institute of Electromagnetic Engineering and Science, vol. 30, no. 3, pp. 236-242, 2019. 